\title{
Utilizar las tecnologías para potenciar las habilidades sociales y comunicativas
}

\author{
Marta AbANADES SÁNCHEZ \\ Universidad Europea de Madrid \\ marta.abanades@uem.es
}

Resumen:

Las empresas y departamentos encargados de la contratación de perfiles, no solo buscan profesionales con formación superior sino personas que posean capacidades, cualidades y habilidades que puedan desarrollar y utilizar dentro de una organización.

Palabras clave: docente; tecnologías; habilidades comunicativas; currículum vitae

How to use technology to enhance communication skills in the classroom

\begin{abstract}
:
Companies and departments in charge of hiring profiles, not only are looking for professionals with training, but also people who have skills and qualities that can be developed in the organization.
\end{abstract}

Key Words: teaching; technology; communication skills; video resume

\section{Referencia normalizada:}

Abadanes Sánchez, M. (2014): Utilizar las tecnologías para potenciar las habilidades sociales y comunicativas. Historia y Comunicación Social. Vol. 19. Núm. Especial Febrero. Págs. 679-688.

Sumario: 1. Introducción. 2. Metodología. 3. Evaluación. 4. Resultados. 5. Posibles Aplicaciones futuras. 6. Referencias Bibliográficas.

\section{Introducción}

Nos encontramos en un panorama desolador para los jóvenes titulados que finalizan carrera universitaria y se encuentran con el desamparo de no poder acceder a un trabajo acorde con su perfil. No solo ponen de manifiesto el no poder ejercer sino también el desconocimiento de las herramientas de acceso al mercado laboral para tener una primera toma de contacto.

Hoy en día no en suficiente obtener en el currículum vitae títulos que abalen los conocimientos teóricos o fundamentados del puesto a cubrir. Esta formación ya no es un valor añadido, sino una obligación. 
Pero además las empresas y departamentos de contratación buscan personas con capacidades, cualidades y habilidades de comunicación para que puedan desarrollar un trabajo de éxito llegando a transformar mediante sus competencias, la visión en realidad.

Los directivos y encargados de la selección y contratación de personal, cada vez son más conscientes de la efectividad del Efecto Hawthorne, experimento social que demostró que el rendimiento dependía más de lo humano (motivación, relaciones interpersonales, participación en el diseño del puesto, autogestión) que lo técnico (colocación, luminosidad, horarios, control) (García-Rincón de Castro Cesar y Hernández Hernández Carlos, 1992:10).

Pero además, los expertos seleccionadores, head-hunters, conocedores de la importancia del factor humano en la organización, expresan lo imprescindible que es que el candidato (junior, intermedio, senior) posea una gran caja de herramientas sociales y comunicativas.

Los recursos utilizados hasta el momento para poder acceder a las empresas, como la carta de presentación, currículum vitae en mano, correo electrónico a la propia persona o departamento, ya no son métodos suficientes para un primer acercamiento.

La entrevista por excelencia ha sido el anclaje utilizado para conocer al candidato; está entrevista de selección es un forma de comunicación de expectativas previas a la experiencia real en el desempeño del puesto dentro del marco de la organización, debido al contenido de la conversación, que gira en torno a las dudas sobre responsabilidades, beneficios, pagas, relaciones etc..) (Jablin 1987 citado por: Palací y Peiró, 1995a: 59).

Las empresas de selección intentan captar al mejor candidato que se ajuste a la oferta de la empresa tanto a nivel nacional o internacional, donde su socialización y entrada a la organización sea prácticamente automática y en muy poco tiempo funcione como uno más de la plantilla.

Mediante la selección, las organizaciones y empresas buscan la congruencia entre las habilidades y destrezas del individuo y los requerimientos del puesto, así como los deseos del individuo y el clima y cultura de la organización. (Palací y Peiró, 1995b: 61).

Pero las herramientas de acercamiento conocidas para los candidatos están en desuso o por el contrario, no llegan a recibir contestación alguna.

La carta de presentación y currículum vitae enviado por correo ordinario, acercarse a la propia sede de la organización para dejar el currículum con la posibilidad de obtener información para una posible toma de contacto, las cuentas de correo electrónico destinadas a recibir currículum actualmente desbordadas diariamente, estás y otras opciones quedaron en un segundo plano para poder obtener un primer acercamiento con la empresa, pero aun así, tampoco las podemos descartar como vía, o método de 
acercamiento. La propuesta, es transmitir o enseñar otras vías de acceso que puedan utilizar para lograr ese primer acercamiento, por ejemplo: el video currículum.

El video currículum ofrece la posibilidad de obtener perfiles sin importar la distancia física para elegir al candidato más idóneo para el puesto, pero además se tiene la posibilidad de poder ver y saber el manejo de las habilidades comunicativas por parte del candidato.

En la vida ordinaria, social y empresarial, se aprecia que las personas que triunfan no son siempre las más inteligentes, en función de las puntuaciones que obtuvieron en los test, o por resultados académicos, sino porque tienen una forma de actuar especial basada en el control de sus emociones (Olleros, 2005: 34).

Se busca en los candidatos esa capacidad para poder comunicarse de manera asertiva, con facilidad para relacionarse y socializarse en el menor tiempo posible dentro de la organización, cualidades como la adaptabilidad, flexibilidad, liderazgo, resolución de conflictos, capacidad de negociación, trabajo en equipo, auto-motivación y por excelencia una de las más básicas y demandadas como: las habilidades sociales y comunicativas.

La herramienta del video currículo refleja la destreza que posee el candidato ante la cámara, transmitiendo confianza en uno mismo, destacando vestimenta, facilidad de palabra, el orden a la hora de priorizar los datos y la capacidad comunicativa que posee el candidato para transmitir la información de su perfil. En las últimas décadas, la educación superior aspira a ser una educación integral, en la que la labor del profesor incluya tanto la docencia como la orientación, atendiendo siempre a la diversidad de características y peculiaridades de los estudiantes (Sebastián y Sánchez, 1999). Con todo lo aportado, no podemos olvidar nuestra función como docente y el papel tan importante a desempeñar con los alumnos para cumplir con las expectativas que exige el mercado.

Hay un proceso histórico de aumento de las exigencias que se le hace al profesor o docente, pidiéndole asumir cada vez mayor número de funciones y responsabilidades con sus alumnos. Actualmente un profesor no puede afirmar que su tarea se reduce simplemente a dar sus clases, sino que, además de su materia, se le pide y exige que sea un facilitador del aprendizaje, pedagogo eficaz, organizador del trabajo en grupo y que además de atender la enseñanza, cuide el equilibrio psicológico y afectivo de sus alumnos, integración social etc. (Esteve, 2009a: 22).

Los avances tecnológicos en el área de educación, se ponen de manifiesto para que los docentes y profesionales en los centros educativos, opten por nuevas metodologías más activas, innoven en sus materias y utilicen un nuevo método de enseñanza aprendizaje mediante el uso de las tecnologías. El docente tecnológico será un mediador, que trabajara de manera transversal con su equipo docente, utilizara el error como fuente de aprendizaje y fomentará que el alumno tenga su propia autonomía en el proceso de enseñanza aprendizaje. (Muñoz, 2003: 5). El hecho de que el docente tenga una actitud más positiva o negativa a la hora de desarrollar su función y 
acompañarla de entornos tecnológicos está fuertemente condicionada por las siguientes propuestas: la disponibilidad del docente para una formación permanente en todos estos recursos, la preparación que posee para el uso de la tecnología, y el poder tener un espacio disponible acondicionado para poner en práctica las actividades y tareas (Gisbert Cervera, Mercé. 1999: 2). El papel del tutor, también ha sufrido cambios y para poder conseguir unos estándares de calidad en la enseñanza, este profesional tendrá que realizar sesiones con un seguimiento más exhaustivo y personalizado con sus alumnos. Los alumnos también requieren de está atención tan inmediata y activa, para ellos la evaluación continua y la retroalimentación que pueda recibir es el input de las metas conseguidas.

Los cambios en la sociedad y el sistema educativo han llevado a una cierta confusión acerca de la función tutorial, pero parece claro que la labor del tutor consiste en relacionarse con el tutelado con el objetivo de desarrollar sus capacidades y la formación de su personalidad (López, 2003). Considerando todas las aportaciones y argumentaciones, se podría afirmar que, formación, habilidades y tecnología son tres palabras que deben ir unidas y tienen que estar presentes tanto en la práctica del profesor o docente como en el currículum del alumno.

Con la experiencia que se detallara a continuación, se pone de manifiesto poder trabajar dentro del aula fomentando las habilidades comunicativas, teniendo en consideración toda la documentación y formación propiamente de la materia $\mathrm{y}$ además utilizando como recurso las tecnologías (el video currículum), poniendo en sus manos un recurso más para el acercamiento y primera toma de contacto con la empresa.

Para los alumnos, la teoría impartida dentro del aula ya no es suficiente, es necesario buscar y fomentar nuevas maneras de innovar en el proceso enseñanza y aprendizaje, que puedan aprender no solo de manera individual sino también colaborativa para poder aprender y desarrollar competencias que más tarde utilizaran en los entornos de trabajo.

Para los docentes y profesionales de la educación, uno de los objetivos o metas, en mejorar la calidad de la educación y desarrollar la sociedad del conocimiento. Con todo lo abordado podemos llegar a la conclusión de que la sociedad del conocimiento, la calidad del sistema educativo y la formación del profesorado, se han convertido en ejes claves para obtener talento y desarrollar capital humano, no solo por parte de los entornos y sistemas educativos sino también por parte de las empresas y organizaciones.

Como docentes debemos aceptar el cambio social, a ser capaces de modificar nuestros trabajos las veces que sean necesarias en nuestro recorrido profesional y tener la competencia social de poder asumir y enfrentarnos a situaciones desconocidas y en ocasiones conflictivas (Esteve, 2009b: 23). 


\section{Metodología}

Para la utilización y puesta en práctica de la herramienta del video currículum, la muestra utilizada, fueron alumnos de último curso académico en uno de los grados de la Facultad de Ciencias Sociales. A pesar de que los alumnos estuvieran en su último curso académico, no poseen experiencia profesional en su sector, por lo que la práctica a priori les parece interesante para poder conseguir nuevos métodos de acercamiento al mercado laboral. En la propia materia ya habían podido ver procesos de selección y la importancia de la aplicación de las técnicas de comunicación tanto en su vida personal como profesional. La puesta en práctica se llevó a cabo poco antes de finalizar la materia, ya que la intención es que tuvieran los conocimientos teóricos suficientes con respecto a los procesos de selección y que también hubieran practicado las técnicas de comunicación en diferentes entornos.

Para el desarrollo de la práctica se siguió la siguiente estructura gráfica que representa el modelo enseñanza aprendizaje que se utilizó para que los alumnos pudieran aplicar los conocimientos teóricos y prácticos dentro del aula:

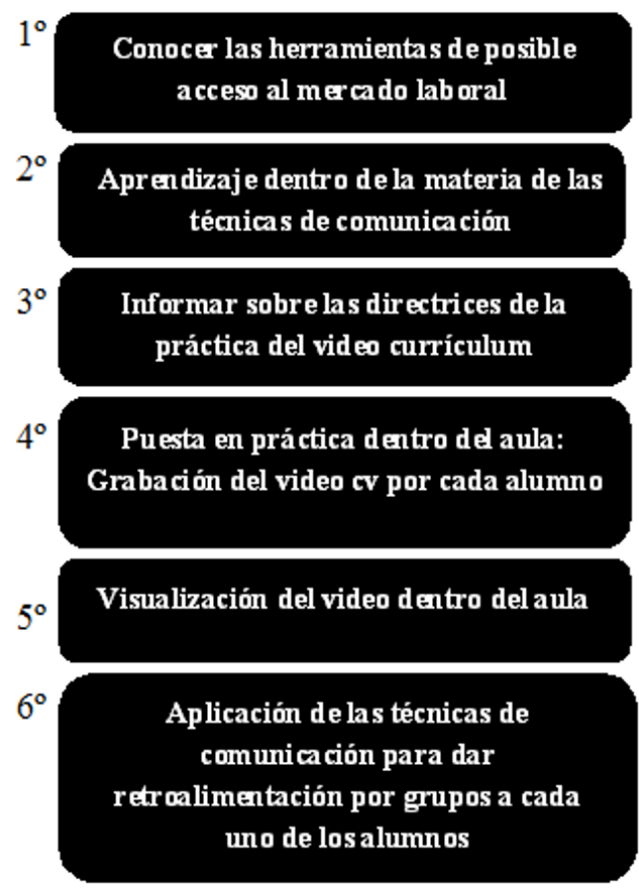

Los puntos 1 y 2 (Conocer las herramientas de posible acceso al mercado laboral y Aprendizaje dentro de la materia de las técnicas de comunicación) se desarrollan dentro de manera teórica dentro del aula. Los alumnos además de conocer las técnicas de comunicación existentes (feed back, escucha activa, crítica constructiva, etc.,) 
las ponen en práctica en diferentes entornos (familiar, amistad, académico...,). Como sugerencias para exponerse delante de una cámara y hablar de su perfil se les ofrecen las siguientes sugerencias:

Para empezar es necesario conocer a que empresa va dirigido, esto ayudara o nos dará pistas de como transmitir la información y cuál puede ser más relevante para la organización.

A continuación, en el punto 3, será necesario hablar de manera clara y pausada, detallar de manera organizada el perfil, los 30 primeros segundos del video son fundamentales para el entrevistador, será necesario intentar captar la atención para que prosiga viendo el resto del video, uno de los valores añadidos en poder enfatizar en las fortalezas y habilidades de uno mismo, también ayudara el ser original y tener una actitud positiva, sonreír, ponérselo fácil al entrevistado mostrando nuestro correo electrónico o página web, para que pueda acceder a nuestros datos y poder conocer mayor información de nuestro perfil, por último el mensaje debería de responder las siguientes preguntas: ¿Quién soy, que hago y cómo trabajo?.

Por último, los alumnos que lo consideren oportuno, según a la empresa u organización o conociendo la oferta en concreto, podrá realizar la presentación en otro idioma.

La puesta en marcha correspondiente al punto 4 dentro del aula es sencilla, suficiente con utilizar una cámara de video o cualquier otro medio tecnológico donde recoge de manera ininterrumpida uno a uno la argumentación de cada uno de los alumnos. Una vez que todos los alumnos han defendido su perfil delante de la cámara, se pondrán en práctica las técnicas de comunicación aprendidas dentro del aula.

En los puntos 5 y 6 , todos los alumnos visualizaran el video donde se mostrara la grabación de cada uno de sus compañeros y en grupos de 4 o 5 alumnos, a continuación los grupos realizaran un feed back y/o retroalimentación a cada uno de los alumnos. En este punto aprovechamos toda la información que el alumno conocer sobre la teoría y práctica de las técnicas de comunicación, con esta retroalimentación ofrecida a cada uno de los alumnos, serán consciente de como poder mejorar su presentación, como se muestran al público, la utilización de los elementos verbales y no verbales, serán conscientes de las palabras, pausas etc., que han utilizado, no solo serán consientes de como poder mejorar sino también de como se muestra, como transmite el mensaje y como le ven sus iguales.

Todos los grupos formados, realizaran este mismo ejercicio con cada uno de los alumnos que realizo su video currículum, por lo que cada alumno se llevara tantas retroalimentaciones y/o críticas constructivas como grupos se hallan formado dentro del aula. 


\section{Evaluación}

La evaluación que se realiza en las prácticas realizadas dentro del aula, tienen una especial importancia tanto para el profesor como para el alumno, ambos tienen que sentir que los objetivos se han cumplido. Por un lado el profesor con la puesta en marcha del modelo enseñanza aprendizaje utilizado en el aula, como el alumno por el conocimiento de una posible herramienta para un primer acercamiento con la empresa además de manifestar y poner en práctica con sus compañeros las técnicas de comunicación. Para la evaluación de esta práctica, no solo se tendrá en consideración la calificación numérica del profesor o docente, sino que se tendrán en consideración en la puntuación final, de manera cualitativa y cuantitativa las retroalimentaciones y evaluaciones por cada uno de los grupos a cada alumno.

De esta manera, los alumnos, al colaborar en las puntuaciones finales de cada uno de sus compañeros, podrán sentirse responsables y participes de su propio aprendizaje.

Otro de los valores que tiene la utilización de medios tecnológicos, es que en el momento de las retroalimentaciones por cada uno de los grupos, es que se pueden volver a ver las grabaciones de los alumnos para rectificar o no comentarios o aportaciones al respecto.

Con esta metodología se pretende también practicar el aprendizaje colaborativo dentro del aula además de fomentar una experiencia social y académica.

\section{Resultados}

Con la utilización de esta práctica, los alumnos se muestran más participativos, colaborativos en cada una de las sesiones que se desarrolló la práctica. Se puede resaltar que se cumplieron los objetivos propuestos, tanto la práctica de las técnicas de comunicación dentro del aula con cada uno de sus compañeros como también, dar a conocer otra herramienta de acceso al mercado laboral como el video currículum para poder conseguir una primera toma de contacto con el mundo laboral.

Para finalizar a los alumnos se les paso un cuestionario cuantitativo y cualitativo para conocer la valoración que para ellos había representado esta práctica.

La media cuantitativa fue de 7,6 , esta cifra nos indica que los alumnos la valoran de manera notable y como resultados cualitativos, los alumnos respondieron los siguientes comentarios y sugerencia a la pregunta sobre los valores añadidos que tiene está práctica.

Se corrigen errores que cometemos, el feed back grupal permite hacer una labor de corrección más eficiente(A-1) 
Práctica positiva, te conoces a ti mismo y la forma en que te perciben los demás (A-2)

Puedes ver tus fallos y los de tus compañeros de una manera constructiva para así aprender de ellos (A-3)

Te ayuda a quitarte la vergüenza (A-4)

La persona adquiere un manejo directo en habilidades comunicativas al poder visualizarse y corregir errores que de otro modo resultaría altamente dificultoso (A-8)

Se conoce de manera directa nuestra manera de hablar al público y valorar nuestro lenguaje corporal (...) estamos valorando si el tono es adecuado, si utilizamos el lenguaje verbal y no verbal, comunicación(A-14)

Original y diferente (A-15)

Te quita timidez, te ayuda a expresarte, te prepara para el futuro (A-17)

Ahorra tiempo al seleccionador, también la posibilidad por nuestra parte de repetir la prueba y tiene un bajo coste para las empresas y profesionales que se dedican a la selección y contratación (A-20)

En la sociedad actual cada vez está más demandada (A-24)

Estas respuestas son algunos comentarios que escribieron los alumnos, donde justifican las fortalezas de la realización de esta actividad, para no solo poner en práctica las habilidades y manejar otra herramienta de acceso al mercado laboral, sino también para poder corregir errores que se pueden cometer en la comunicación verbal y no verbal y fundamentalmente a ganar confianza en uno mismo en el momento de exponerse para poder venderse además de la utilización de tecnologías para entrar en el mercado laboral. Los alumnos se sienten más motivados para poder enfrentarse a una entrevista laboral o adentrarse en el mercado laboral. Por todas las respuestas obtenidas en cada una de las cuestiones, los alumnos consideran que la herramienta es útil y la manera de aprender diferente y enriquecedora.

Con esta metodología se intenta no solo fomentar el aprendizaje colaborativo dentro del aula, sino que los alumnos sean capaces de engordar su aprendizaje en las habilidades comunicativas. No solo se busca que los alumnos aprueben la materia para el expediente, sino también con el valor añadido de que puedan utilizar las tecnologías para su currículum vitae, tecnologías tan demandadas en estos momentos por nuestra sociedad para buscar diferenciarse en el mercado con herramientas diferentes que puedan llamar la atención de los seleccionadores y empresas encargadas de la contratación. Además los alumnos con esta práctica fomentan la posibilidad de mostrarse de manera asertiva y saber venderse mostrando fortalezas y valores es unos minutos ante la cámara. 
Los alumnos valoran el poder poner en práctica dentro del aula los contenidos teóricos de la materia y poder aprender no solo por uno mismo sino también de las aportaciones de los compañeros y retroalimentación recibida dentro del aula.

\section{Posibles aplicaciones futuras}

Los docentes reconocen el tener que innovar dentro del aula para una mejora del proceso de enseñanza aprendizaje utilizado con sus alumnos, para conseguir mayor entusiasmo y motivación en la participación de las clases. Considerando los resultados y los atractivo para los alumnos el poder poner de manifiesto la teoría de manera práctica dentro del aula y utilizarla para su currículum vitae, esta actividad se volverá a repetir, pero con un planteamiento diferente. Se buscara poder compartir esta práctica con otras materias similares dentro de la titulación y poder buscar semejanzas de actividades con otros profesores.

Los docentes y profesionales educativos deben asumir nuevos retos tecnológicos y metodológicos en pleno auge para la sociedad, posicionándose como un profesor con un rol flexible, con adaptabilidad al cambio, como mediadores no solo dentro del aula con nuestros alumnos sino también con nuestros compañeros y equipos docentes.

Es necesario trabajar no solo la motivación, el entusiasmo, las ganas por enseñar $\mathrm{y}$ aprender en los diferentes entornos, tanto dentro como fuera del aula. La nueva propuesta para los docentes será trabajar de manera pro activa, más transversal y multidisciplinar, para poder trasladar no solo unos conocimientos teórico - prácticos sino también unos valores humanos que permitan al alumno desarrollarse no solo como buenos profesionales sino también como personas. Profesores y alumnos deben de seguir apropiándose de las herramientas tecnológicas para sacar el máximo partido posible a los contenidos y conocimientos dentro del aula. Sera necesario que podamos transformar nuestra visión en realidad para seguir enseñando, transmitiendo valores y ayudarles a enfrentarse a las incertidumbres que se van a encontrar nuestros alumnos en la sociedad que están viviendo para que puedan superar con éxito sus miedos y consigan cumplir sus sueños.

\section{Bibliografía}

6.1 Libros:

GARCÍA-RINCÓN DE CASTRO, César; HERNÁNDEZ FERNÁNDEZ, Carlos (1992): Gafas, brújulas y herramientas: guía práctica para desarrollar tus habilidades sociales en la empresa. Ed. Homo Prosocius. Madrid. 
JABLIN, F.M. (1987). Organizational Entry, Assimilation, And Exit. In F.M. Jablin, L.L. Polman, K.H. Roberts y L.W. Porter (Eds): Handbook of Organizational Comunication: An Interdisciplinary Perspective, 679-740, Newbury Park, CA: Sage.

JEFFREY, A. Kelly. (1992): Entrenamiento de las habilidades sociales. Ed: Desclee De Brouwer.

OLLEROS IZARD, Manuel (2005): El proceso de Captación y selección de Personal. Gestión 2000. Barcelona.

PALACÍ, F. José y PEIRÓ, J. María. (1995): La incorporación a la empresa. Promolibro. Valencia.

6.2 Artículos en publicaciones web:

ESTEVE, José Manuel (2009). La profesión docente ante los desafíos de la sociedad del conocimiento. Aprendizaje y desarrollo profesional docente, 17 - 27. Disponible en: www.oei.es/metas2021/APRENDYDESARRPROFESIONAL. [05/09/2013]

GISBERT CERVERA, Mercé (1999). El profesor del siglo XXI: de transmisor de contenidos a guía del ciberespacio.

www.tecnologiaedu.us.es/nweb/htm/pdf/203.pdf. [06/09/2013]

LÓPEZ ORTEGA, A (2003): La tutoría en la Universidad. Comunicación en el Primer Foro Institucional de Tutoría Académica, celebrado el 30 de octubre de 2003, Guadalajara, México.

MUÑOZ, Ricardo Fernández (2003). Competencias profesionales del docente en la sociedad del siglo XXI. In Organización y gestión educativa: Revista del Fórum Europeo de Administradores de la Educación (Vol. 11, No. 1, pp. 4-7) CissPraxis. http://dialnet.unirioja.es/servlet/articulo?codigo $=292590$. [11/09/2013]

SEBASTIÁN RAMOS, A. \& SÁNCHEZ GARCÍA, M. F (1999): La función tutorial en la universidad y la demanda de atención personalizada en la orientación. Educación XXI: Revista de la Facultad de Educación, 2, 245-264. http://www. upla.cl/educacion/web/wp-content/uploads/daura-revista-orientacion-primer-semestre-2011.pdf. [06/09/2013]

\section{La autora}

Marta Abanades Sánchez licenciada en psicopedagogía y diplomada en educación social, con varios programas de postgrado: Máster Internacional en Coach Profesional y Ejecutivo (IEC), Máster en Recursos Humanos (CEREM) y Máster Universitario en Formación al Profesorado (UEM). Profesora asociada al Dpto. Educación y Desarrollo Profesional en la Universidad Europea de Madrid impartiendo materias como: Habilidades Comunicativas, Habilidades Directivas y Dirección de Personas. 